\title{
Orthorexia nervosa vs. healthy orthorexia: relationships with disordered eating, eating behavior, and healthy lifestyle choices
}

\author{
Hana Flynn Zickgraf ${ }^{1}$ [D . Juan Ramón Barrada²
}

Received: 7 April 2021 / Accepted: 28 June 2021 / Published online: 17 July 2021

(c) The Author(s), under exclusive licence to Springer Nature Switzerland AG 2021

\begin{abstract}
Background Orthorexia nervosa ( $\mathrm{OrNe}$ ) is a pattern of disordered eating behavior characterized by excessive preoccupation with overvalued ideas about healthy eating. Healthy orthorexia (HeOr) refers to a non-pathological interest in healthy eating and nutrition. Despite converging evidence that OrNe is correlated with measures of psychopathology and personality traits, there is relatively little empirical data relating OrNe and $\mathrm{HeOr}$ to eating and health-promoting behavior.

Methods Aim 1: exploratory structural equation modeling was used to evaluate the factor structure of an English-language version of the Teruel Orthorexia Scale, the only measure of OrNe that also assesses HeOr in two samples (Yoga practitioners, $N=469$, and Mechanical Turk workers, $N=453$ ). Aim 2: conducted in the Mechanical Turk sample, partial correlation and linear regression analyses were used to evaluate the relationships of OrNe and HeOr with other symptoms of, and clinical impairment from, disordered eating, food choices, diet quality, body mass index (BMI), nutrition knowledge, alcohol/ tobacco/vaping use, and sedentary behavior.

Results The two-factor structure of the TOS was replicated. HeOr and OrNe had opposing relationships with measures of disordered eating behavior and distress, food choices, diet quality, and nutrition knowledge. HeOr was negatively related to BMI, whereas OrNe was positively related to substance use and sedentary behavior.

Conclusions $\mathrm{OrNe}$ and $\mathrm{HeOr}$ are distinct latent constructs, with the latter reflecting non-pathological behavior. Only OrNe is related to elevated disordered eating and clinical impairment. Despite involving obsessions and compulsions related to healthy eating, OrNe was associated with relatively unhealthy eating and other lifestyle behaviors.

Level of evidence Level V, descriptive cross-sectional study.
\end{abstract}

Keywords Orthorexia nervosa $\cdot$ Eating disorder $\cdot$ Food choice $\cdot$ Nutrition $\cdot$ BMI

\section{Introduction}

Orthorexia nervosa $(\mathrm{OrNe})$ is a proposed new eating disorder diagnosis characterized by rigid preoccupation with a perceived "healthy" diet and overvalued nutritional beliefs leading to psychosocial impairment and/or weight and nutritional consequences [1]. OrNe is distinct from healthy orthorexia (HeOr), which is defined as healthy interest in diet, healthy eating behavior, and eating healthily as part of one's identity [2]. It is also distinct from symptoms of currently

Hana Flynn Zickgraf

zickgraf@southalabama.edu

1 Department of Psychology, University of South Alabama, 75 S. University Blvd., Mobile, AL 36688, USA

2 Department of Psychology and Sociology, Universidad de Zaragoza, Teruel, Spain recognized restrictive eating disorders including anorexia nervosa and bulimia nervosa (i.e., shape/weight eating disorders), and avoidant restrictive food intake disorder (ARFID) $[3,4]$. Although there is still ongoing discussion of the definition and clinical utility of the OrNe construct, there is agreement in the field on the need to better understand the psychopathology, comorbidity, and physical morbidity associated with this disorder $[5,6]$.

The two most commonly used measures of OrNe (excluding those with severe psychometric limitations) are the Eating Habits Questionnaire (EHQ) and the Dusseldorf Orthorexia Scale (DOS) [7, 8]. The EHQ was initially tested in two samples from the USA. The DOS was initially validated in a German sample. Both the EHQ and the DOS present some contradictory results in terms of their internal structure.

The EHQ was initially designed and validated to measure three factors: Problems associated with healthy eating, 
knowledge of healthy eating, and feeling positively about healthy eating [7]. Some studies could not replicate this structure [9-13]. Even the interpretation of the EHQ is not clear. It has a group of items that load together across differing factor solutions (variously labeled knowledge of healthy eating, rigid eating behaviors, behaviors, and healthy eating cognitions) and appear to tap $\mathrm{HeOr}$ [4, 9-12]. However, the measure was not intended by its original authors to capture non-pathological aspects of interest in healthy eating [7].

With respect to the DOS, data did not support the presence of a single dimension for the German, English [8, 14], French [15], and Chinese [16] versions of the scale. However, in the Spanish version, a unidimensional model offered an excellent fit [17]. Meule et al. (2020) also obtained a good fit for the unidimensional solution of the original German DOS [11].

Of the existing measures of OrNe, only the newer Teruel Orthorexia Scale (TOS) was designed to also assess HeOr. The TOS is currently validated only in Spanish [18]. OrNe and $\mathrm{HeOr}$ are clearly distinct constructs: prior publications have reported moderate correlations of $r=0.35,0.39,0.46$, and 0.51 [18-21], yet in regressions or partial correlation analyses controlling for the other subscale, OrNe and $\mathrm{HeOr}$ have different patterns of correlations with motivations for food choice, personality, disordered eating symptoms, body image concerns, and symptoms of other psychopathology $[18,19]$. In general, OrNe is positively associated with maladaptive traits, whereas $\mathrm{HeOr}$ has a null or negative relationship with the same traits.

The current OrNe literature has several notable gaps beyond, although related to, the need for better psychometrics. Although OrNe defined as reflecting a rigid preoccupation with overvalued dietary rules, relatively few studies have reported on the relationships between OrNe symptoms and actual eating behavior. To date it is still unclear whether people with symptoms of orthorexia nervosa eat diets that appear to be "healthier" (e.g., containing more plant-based and fewer processed foods, and/or containing less calorically dense, lower-fat, and lower-sugar foods) or more rigid and restrictive than those without OrNe symptoms. Two studies on this topic, both of which used the EHQ (although with differing factor structures and number of subscales) to explore the relationship between $\mathrm{OrNe}$ symptoms and diet quality, yielded largely consistent results. Zickgraf and colleagues [4], using the developers' originally proposed three-factor structure, found that the subscale of the EHQ tapping OrNe-related distress and impairment ("Problems"/"Problems associated with healthy eating") was positively related to higher self-reported fruit and vegetable intake, but also positively related to higher self-reported dessert and snack food intake. Of note, these results were only apparent when controlling for the EHQ "Beliefs" subscale that assesses healthy eating intentions and behaviors (which these authors interpreted as capturing HeOr) [4]. Halim and colleagues [10] found similar results, despite not interpreting the Beliefs items as reflecting HeOr. They reported that only the so-called "Rigid eating behavior" scale (made up of "Beliefs" items that appear to reflect HeOr) was related to higher odds of reporting healthy eating behaviors and lower discretionary food intake [10]. However, in a German sample scoring below the published DOS clinical cut-score of 30 for probable OrNe, higher scores were associated with healthier eating according to German nutritional recommendations [22]. Because OrNe and HeOr appear to have opposite relationships with food intake, particularly of foods considered to be relatively "unhealthy," suppression effects may obscure the true relationship between OrNe and food intake. It may be necessary to statistically adjust for $\mathrm{HeOr}$ in analyses exploring the impact of OrNe on eating behavior. Direct comparisons of prior findings on the eating behavior correlates of $\mathrm{HeOr} / \mathrm{OrNe}$ are complicated by a lack of clarity about what the EHQ and DOS are measuring, a problem with the validity of existing measures of $\mathrm{HeOr}$ and OrNe. Given the history of measurement validity problems in the orthorexia field, continued research on the psychometrics of measures, including the TOS, is needed.

To date, the orthorexia literature has been limited by (1) the lack of valid measurement tools for participant selfreport of OrNe and $\mathrm{HeOr}$, and (2) the lack of studies exploring disordered eating, impairment, actual eating behaviors and food choices, and health behaviors in individuals with OrNe symptoms $[4,10,22]$. Before the field can come to a consensus that OrNe reflects a psychological illness, there is a need for research aimed at identifying and isolating signs of clinical impairment and distress linked to this set of behaviors, and a clear distinction must be made between this construct and HeOr. We should be sure that the OrNe construct does not over-pathologize an interest in healthy eating; even a strong, focused or restricted interest is not inherently problematic. Obsessive thoughts or compulsive behaviors become problematic when they cause distress or lead to impairment.

The current study has two aims which address both identified gaps in the orthorexia literature. The first aim is to establish the factor structure and convergent validity of the English TOS in two samples, one including yoga practitioners [a population that has previously been found to be at higher risk for OrNe, (e.g., 20)] and the other an unselected sample from Amazon's Mechanical Turk (MTurk). We expect to replicate the published two-factor solution of the Spanish TOS. We evaluated convergent validity using another measure of orthorexia nervosa, the DOS. We predicted that, controlling for TOS-OrNe, TOS-HeOr would be minimally correlated with the DOS, whereas controlling for TOS-HeOr would not attenuate the predicted strong-moderate correlation between the DOS and TOS-OrNe. 
The second aim is to explore the hypothesized differential relationships between the two TOS subscales and measures of weight/shape disordered eating, eating disorder clinical impairment, diet quality/eating behavior, nutrition knowledge, problematic alcohol use, smoking, vaping, and exercise. Consistent with other studies, we expect to find a moderate relationship between the TOS subscales and measures of weight/shape disordered eating and clinical impairment from disordered eating, but hypothesize that partial correlation analyses will demonstrate that this relationship is specific to OrNe whereas $\mathrm{HeOr}$ is unrelated or inversely related to weight/shape disordered eating and disordered eating clinical impairment $[4,23]$. We hypothesize that a normative interest in healthy eating measured by the TOS-HeOr scale will be positively associated with other indicators of a generally healthy lifestyle in analyses statistically adjusting for levels of OrNe. Variables of interest include less problematic drinking, less nicotine use, and less sedentary behavior, in addition to eating behavior and nutrition knowledge that generally conforms to current scientific standards of healthy eating. We hypothesize that the TOS-OrNe will show the opposite patterns of relationships with lifestyle variables and nutrition knowledge, in addition to being negatively related to overall diet quality, and positively related to intake of both fruits and vegetables and discretionary foods [4].

\section{Methods}

\section{Participants and procedure}

\section{MTurk sample}

Participants were 502 adults recruited on Amazon's Mechanical Turk (MTurk) in May 2020. MTurk is a platform where researchers and businesses post tasks that can be completed online, including research surveys. Participation was restricted to individuals living in the United States and to individuals older than 18. Participants in this study were paid $\$ 6$ for completing a series of surveys that took approximately 30-60 min to complete. Participants who failed one or more embedded validity checks $(n=25)$, or provided incomplete data on the TOS $(n=24)$ were excluded yielding a final sample of 453. Participants in the final sample were majority White (71.2\%), with $40.8 \%$ identifying as cisgender women, $54.1 \%$ as cisgender men, and $5.1 \%$ as minority genders (i.e., transgender men and women and nonbinary individuals). $64 \%$ of the sample had a 4 -year college degree or higher education, and the median annual household income was US \$50-60,000. See Table 2 for all sample descriptives. The IRB at the University of Chicago approved all study procedures; Participants indicated their informed consent when agreeing to participate.

\section{Yoga sample}

Data came from Domingues and Carmo (2020), who included their original data as supplementary material [20]. Four-hundred sixty-nine yoga practitioners, $84 \%$ of whom were women, $57 \%$ of whom ranged from 35-55 years of age, and who were highly educated $(85.7 \%$ with complete college or university education or more) completed the online questionnaire. Participants were recruited by emails sent to yoga schools in Portugal, UK, and USA, asking to share the link of the study with their members; the link was also posted on yoga groups on Facebook.

\section{Measures}

\section{Teruel Orthorexia Scale (TOS; Barrada and Roncero, 2018)}

This scale assesses orthorexia with two separate dimensions: OrNe (eight items) and HeOr (nine items). Responses are provided on a four-point scale ranging from $0=$ Completely disagree to $3=$ Completely agree. Scores by dimension were computed as the sum of the item responses. Although Dominguez and Carmo (2020) used an English translation of the TOS [20], a new and improved version was used here. Starting with that initial translation, both authors discussed and agreed with that final version. The wording of six items were slightly changed at the suggestion of the first author, who is a native English speaker, because the original items reflected direct translations that are not consistent with standard English (e.g., the item "I believe that the way I eat is healthier than that of most people" was changed to "I think that my way of eating is healthier than that of most people"). This new version was applied in the MTurk sample, while for the yoga sample the initial English version was used. The full text of the improved and validated (MTurk sample) TOS-English is available as an Appendix.

\section{Dusseldorf orthorexia scale—English (DOS; [14])}

The DOS is a ten-item self-reported questionnaire to measure orthorexic eating behavior and related thoughts and feelings (e.g., "If I eat something I consider unhealthy, I feel really bad"). Answers are scored on a four-point Likert-scale from $1=$ this does not apply to me to $4=$ this applies to me . In the current sample $\alpha=0.90$.

\section{Eating disorder examination questionnaire-7-item form (EDE-Q [24])}

The 7-item short form of the EDE-Q [25] is a validated measure of behaviors and cognitions related to weight/ 
shape disordered eating. The EDE-Q is scored by taking the mean of all seven items, which assess the extent to which individuals endorse restrictive eating behavior to influence weight and shape, dissatisfaction with weight and shape, and excessive influence of weight and shape on self-image. The EDE-Q global score ranges from 0-6. In the current sample $\alpha=0.92$.

\section{Clinical impairment assessment-eating (CIA-E; $[26,27])$}

The original CIA is a validated measure of psychosocial impairment due to restrictive disordered eating. The CIA has been modified to assess the psychosocial impact of disordered eating behavior that is not related to weight and shape concerns (e.g., ARFID, orthorexia nervosa) by removing references to caloric restriction, excessive exercise, and compensatory behaviors from the instructions [27]. The CIA includes 16 items rated on a $0-4$ scale including "to what extent have your eating habits... made it difficult to concentrate," "...made you feel ashamed of yourself" and "... made it difficult to eat with others." Total scores is the sum of each item score, yielding a score ranging from 0-48. In the current sample $\alpha=0.97$.

\section{Rapid assessment of eating for patients—short form (REAPS [28])}

The REAPS is a brief screening measure for dietary quality that has shown convergent validity with food frequency questionnaires and dietary recall interviews [29]. Thirteen items assess the frequency of behaviors like skipping breakfast, eating fast food, eating fewer than two daily servings of fruit and of vegetables, eating fried foods, and eating more than $8 \mathrm{oz}$ of red meat per day, yielding a total summed score ranging from 13-39, with higher scores indicating better diet quality. In the current sample $\alpha=0.76$.

\section{Eating behaviors}

[30] Participants reported their typical daily intake "on an average day" of fruits, vegetables, dairy products, starches/ grains, protein-containing foods, snacks, and desserts on a $0-10$ scale, ranging from 0 servings to $10+$ servings. A serving was defined as $1 / 2$ cup of the food. Proportion of daily intake accounted for by discretionary foods and of fruits/ vegetables was computed by summing reported servings of fruits and vegetables, and servings of snacks and desserts, and dividing the result by the total number of reported food servings. Higher values for each measure indicate a greater proportion of total food intake accounted for by discretionary foods and fruits/vegetables, respectively. This measure has been used in a prior study of eating behavior and orthorexia nervosa [4].

\section{General nutrition knowledge questionnaire-revised [GNKQ-R; 31]}

The GNKQ- $\mathrm{R}$ is an 88-item measure tapping awareness of dietary recommendations, healthy eating, and the impact of eating behavior on health and health conditions. The GNKQ was found to be reliable and sensitive to change with nutrition education, as well as externally valid, with dietetics students, participants with more education, and participants with better physical health status scoring higher [31]. Because the GNKQ was developed in the UK, several items that referred to UK-specific dietary recommendations were changed to refer instead to Centers for Disease Control (CDC) recommendations in the United States (i.e., Item 9, "eatwell plate" was change to "MyPlate"). Wording of several other items were changed to describe foods using American, rather than UK, terminology (e.g., Item 7, "biscuits, cakes and pastries" to "cookies, cakes, and pastries," and "rapeseed oil" to "canola oil"). GNKQ-R scores were expressed as percentage of questions answered correctly.

\section{Body mass index (BMI)}

Participants provided their weight and height, which were used to calculate BMI using the following formula: $\mathrm{BMI}=$ weight $(\mathrm{kg}) / \mathrm{height}(\mathrm{m})^{2}$. Eighteen participants who provided heights or weights that yielded unrealistic BMI $(<10)$ were excluded from analyses with BMI as the outcome variable.

\section{Alcohol use disorders identification test (AUDIT [32])}

The AUDIT is a 10-item checklist measuring severity/intensity of problematic drinking with possible scores ranging from 0-40.

\section{Smoking and vaping}

Participants were asked if they currently smoked cigarettes and if they currently vaped tobacco/nicotine products. Those who endorsed smoking and/or vaping were asked on how many of the past 30 days they smoked and/or vaped. Participants who smoked were also asked how many cigarettes they smoked in a typical day.

\section{Sedentary time}

Participants responded to a modified version of the Screen Time portion of the SIT-Q-7D questionnaire [33]. The full SIT-Q-7D is a comprehensive measure of sedentary behavior 
including work, commuting, and leisure activities. The leisure activities portion of the questionnaire was modified by combining several non-screen sedentary leisure activities into a single item and by asking participants to estimate time spent on each sedentary activity in a typical weekday/workday and a typical weekend/non-workday rather than asking participants to estimate their past-week sedentary time dayby-day. Participants reported time spent watching TV, using a computer, playing video games, and "Reading, writing, crafting or other quiet leisure-time activity you do while sitting down" on an 11-point scale ranging from $0=$ no time to $10=$ more than $7 h$. The scale was recoded to units of hours such that $1=0.25,2=0.5,3=1 \ldots 10=8$.

\section{Subjective health status}

Participants responded to the prompt "Would you say that in general your health is..." with anchors $1=$ Poor, $2=$ Fair, $3=$ Good, $4=$ Very good, $5=$ Excellent .

\section{Data analyses}

The internal structure of the TOS scores was analyzed separately for both samples, with exploratory structural equation models (ESEM) [34]. Previous analyses have found that, although the internal structure was clear and theoretically interpretable, secondary loadings cannot be expected to be equal to zero. In cases like this, a confirmatory factor analysis would distort the recovered parameters. For the ESEM models, we used target rotation. Models were analyzed using robust weighted least squares (WLSMV estimator in MPlus). According to conventional cut-offs [35] CFI and TLI with values greater than 0.95 and RMSEA less than 0.06 were indicative of a satisfactory fit. It should be noted that these cut-offs were developed for CFAs with continuous responses, so these values should be interpreted with caution [36].

Zero-order correlations (continuous) and $t$ tests/one-way ANOVA (dichotomous) were computed with the TOS subscales and all study variables. For convergent validity analyses and to explore the relationship between the TOS and weight/shape disordered eating score, partial correlations were computed with each TOS subscale and the DOS/EDE$\mathrm{Q}$, adjusting for the other subscale. Given the predicted moderate correlations between OrNe and the EDE-Q, all other Aim 2 analyses were conducted using multiple regression with the two TOS subscales and the EDE-Q as predictors. Primary study hypotheses involved isolating the independent effect of each TOS subscale from that of the other TOS subscale and from weight/shape disordered eating. Because of the large number of regression analyses (10 dependent variables) and coefficients being interpreted in study hypotheses (three coefficients $\times 10=30$ ), family-wise error rate for interpreting the main effect of the TOS subscales and EDE-Q in Aim 2 regressions was set at $p<0.05 / 30=0.002$.

\section{Results}

\section{Aim 1. Internal structure and convergent validity}

\section{Factor analysis}

ESEM revealed an overall adequate fit in both samples, although with a RMSEA slightly over the cut-off value for the MTurk sample. For MTurk/yoga samples, model fit indices were: $\chi^{2}(103)=395.6 / 261.1$, $p<0.001$, RMSEA $=0.078 / 0.057, \mathrm{CFI}=0.978 / 0.967$, and TLI $=0.971 / 0.957$. Primary loadings (loading of items in their intended factor; see Table 1) were high for both samples, $M_{\mid \text {primary } \lambda \mid}=0.74 / 0.67$. Secondary loadings were small, $M_{\text {Isecondary } \lambda \mid}=0.16 / 0.14$. However, three secondary loadings higher than $|0.30|$ were observed. Of those, in two cases the primary loading was clearly greater than the secondary, but for Item 15 ("I try to convince the people in my life to follow my healthy eating habits") in the MTurk sample the reverse pattern was found. The best marker of HeOr was "I mainly eat foods that I consider healthy", while the best one for OrNe was "Thoughts about healthy eating prevent me from concentrating on other tasks". As predicted, the TOS-En subscales were intercorrelated in both samples. In the MTurk sample, $r=0.60$, whereas in the Yoga sample $r=0.32$.

To maintain consistency with results of prior studies that used the TOS, we chose to retain the original factor solution in Aim 2 analyses. All analyses were re-run with Item 15 included on the OrNe factor, and with Item 15 dropped; results of sensitivity analyses are available on request from the authors. They did not differ in direction, magnitude, or significance of effects from the reported results.

\section{Convergent validity}

See Table 2 for zero-order correlations between the TOS subscales and all study variables. The partial correlation between the DOS and the TOS-OrNe was slightly attenuated, but still large: $r=0.62$. Controlling for the TOS-OrNe, the relationship between the DOS and TOS-HeOr was $r=0.49$. 
Table 1 Item loadings for the Teruel Orthorexia Scale

\begin{tabular}{|c|c|c|c|c|}
\hline & \multicolumn{2}{|c|}{ MTurk sample } & \multicolumn{2}{|c|}{ Yoga sample } \\
\hline & $\mathrm{HeOr}$ & OrNe & $\mathrm{HeOr}$ & OrNe \\
\hline 1. I feel good when I eat healthy food & 0.80 & $-\underline{0.44}$ & 0.60 & -0.02 \\
\hline 2. I spend a lot of time buying, planning, and or/preparing food so my diet will be as healthy as possible & 0.71 & 0.16 & 0.66 & 0.09 \\
\hline 3. I think that my way of eating is healthier than that of most people ${ }^{a}$ & 0.86 & -0.05 & 0.84 & -0.12 \\
\hline 4. I feel guilty when I eat food that I do not consider healthy & 0.15 & 0.45 & 0.00 & 0.72 \\
\hline 5. My social relationships have been negatively affected by my concern about eating healthy food ${ }^{\mathrm{a}}$ & -0.05 & 0.91 & 0.15 & 0.55 \\
\hline 6. My interest in healthy food is an important part of the way I am, of how I understand the world & 0.80 & 0.10 & 0.75 & 0.17 \\
\hline 7. I'd rather eat a healthy food that is not very tasty than a good-tasting food that isn't healthy & 0.60 & 0.29 & 0.55 & 0.19 \\
\hline 8. I mainly eat foods that I consider healthy & 0.93 & -0.10 & 0.85 & -0.12 \\
\hline 9. My concern with healthy eating takes up a lot of my time & 0.22 & 0.72 & $\underline{0.34}$ & 0.57 \\
\hline 10. I am concerned about the possibility of eating unhealthy foods & 0.25 & 0.57 & 0.16 & 0.70 \\
\hline 11. I don't mind spending more money on a food if I think it's healthier & 0.71 & -0.06 & 0.57 & -0.09 \\
\hline 12. I feel overwhelmed or sad if I eat food that I consider unhealthy & 0.12 & 0.71 & 0.00 & 0.82 \\
\hline 13. I would rather eat a smaller portion of healthy food than get full from food that may not be healthy ${ }^{\mathrm{a}}$ & 0.72 & 0.13 & 0.66 & -0.02 \\
\hline 14. I avoid eating with people who do not share my ideas about healthy eating & 0.07 & 0.80 & 0.17 & 0.57 \\
\hline 15. I try to convince the people in my life to follow my healthy eating habits ${ }^{\mathrm{a}}$ & 0.38 & 0.47 & 0.32 & 0.28 \\
\hline 16. If I ever eat something I consider unhealthy, I punish myself for it ${ }^{\mathrm{a}}$ & -0.07 & 0.93 & -0.30 & 0.86 \\
\hline 17. Thoughts about healthy eating prevent me from concentrating on other tasks ${ }^{\mathrm{a}}$ & -0.07 & 0.98 & -0.19 & 0.88 \\
\hline
\end{tabular}

Test statistics and effect sizes are bolded for dichotomous comparisons ( $t$-test and ANOVA)

$\mathrm{HeOr}$ healthy orthorexia, OrNe orthorexia nervosa

${ }^{a}$ Indicates that the item wording was modified from that presented in the original TOS article. Shaded cells indicate the factor where the item theoretically belongs. Loadings in bold indicate unsigned loadings above |30|. Underlined loadings indicate cross-loadings above |30|

\section{Aim 2. Relationships with disordered eating, clinical impairment, eating behavior, nutrition knowledge, and health behavior}

\section{Disordered eating symptoms}

When controlling for the TOS-HeOr scale, the relationship between TOS-OrNe and EDE-Q mean score was similar to the zero-order correlation, $r=0.33, p<0.001$, whereas controlling for TOS-OrNe revealed a small negative relationship between TOS-HeOr and EDE-Q: $r=-0.13, p=0.01$.

\section{Eating disorder impairment, diet quality, food choice, and BMI}

In regression analyses predicting (1) clinical impairment from disordered eating (CIA), (2) diet quality (REAPS), (3) fruit/vegetable proportion, (4) discretionary proportion, and (5) BMI the TOS subscales were entered as predictors, along with the EDE-Q (Table 3). In all five models, TOSOrNe and TOS-HeOr showed the predicted relationships with measures of psychosocial impairment, weight, and eating behavior. TOS-HeOr was negatively related to clinical impairment from disordered eating and discretionary food proportion, and positively related to diet quality and fruit/ vegetable proportion. TOS-OrNe was positively related to eating disordered clinical impairment and discretionary food proportion, and negatively related to diet quality and fruit/ vegetable proportion. Together, the TOS subscales and the EDE-Q predicted almost $60 \%$ of the variance in disordered eating impairment, with effects for OrNe and EDE-Q in the same direction. Conversely, EDE-Q scores were positively related to BMI, whereas both OrNe and $\mathrm{HeOr}$ were independently, negatively related to BMI (though the relationship with OrNe fell above the adjusted significance threshold of $p<0.002$ ), with $12 \%$ of variance explained by these three eating behavior constructs. When controlling for the TOS subscales, EDE-Q score was not a predictor of diet quality or fruit/vegetable and discretionary food proportion.

\section{Nutrition knowledge and health behaviors}

Both TOS subscales were significantly related to nutrition knowledge, with effects in opposite directions: HeOr was associated with higher scores on the GNKQ-R (reflecting greater awareness of current nutrition recommendations and healthy eating techniques) whereas OrNe was linked to lower scores. Only OrNe was related to higher scores on measures of problematic drinking behavior, sedentary hours, or odds of being a current smoker or vape user. EDE-Q score was positively related to nutrition knowledge and to sedentary 
Table 2 MTurk sample and group means, zero-order relationships between the TOS subscales and outcome variables

\begin{tabular}{|c|c|c|c|}
\hline & $M(\mathrm{SD})$ & TOS-OrNe & TOS-HeOr \\
\hline TOS-HeOr & $12.34(5.72)$ & - & 1 \\
\hline TOS-OrNe & $8.42(6.81)$ & 1 & $0.65^{* *}$ \\
\hline DOS & $19.34(9.84)$ & $0.80 * *$ & $0.74 * *$ \\
\hline EDE-Q & $1.55(1.42)$ & $0.33 * *$ & $0.12 *$ \\
\hline CIA & $9.13(11.65)$ & $0.66 * *$ & $0.25 * *$ \\
\hline Diet quality & $29.86(4.54)$ & 0.09 & $0.34 * *$ \\
\hline Fruit/veg & $0.23(0.10)$ & $0.12 *$ & $0.45^{* *}$ \\
\hline Discretionary & $0.12(0.09)$ & $0.33 * *$ & -0.06 \\
\hline Nutrition knowledge (percent correct) & $65.11(17.94)$ & $-0.63 * *$ & $-0.27 * *$ \\
\hline AUDIT screening & $5.17(6.31)$ & $0.47 * *$ & $0.24 * *$ \\
\hline Sedentary time (hours/day) & $11.97(4.65)$ & $0.26 * *$ & 0.05 \\
\hline Subjective health status $(1=$ excellent, $5=$ poor $)$ & $2.38(0.97)$ & $-0.28 * *$ & $-0.43 * *$ \\
\hline $\mathrm{BMI}^{\mathrm{a}}$ & $26.55(6.34)$ & $-0.22 * *$ & $-0.28 * *$ \\
\hline Age & $38.2(11.26)$ & $-0.20^{* *}$ & -0.06 \\
\hline \multirow[t]{2}{*}{ Income } & $6.34(3.24)$ & -0.01 & $0.13 *$ \\
\hline & $N(\%)$ & $\begin{array}{l}t \text { test or one-way ANOVA } \\
M(\mathrm{SD})^{\mathrm{b}}\end{array}$ & \\
\hline Cigarette smoking & & $t(176.9)=4.97, d=0.56^{* *}$ & $t(448)=2.02, d=0.24, p=.04$ \\
\hline Current smoker & $124(27.56 \%)$ & $11.29(0.72)_{\mathrm{a}}$ & $13.23(0.54)$ \\
\hline Non-smoker & $326(72.44 \%)$ & $7.35(0.33)_{\mathrm{b}}$ & $12.01(0.31)$ \\
\hline Vape/E-cigarette use & & $t(125.77)=5.20, d=0.64 * *$ & $t(151.59)=2.24, d=0.30 p=.01$ \\
\hline Current user & $91(20.22 \%)$ & $11.95(0.78)_{\mathrm{a}}$ & $13.64(0.55)$ \\
\hline Non-user & $359(79.78 \%)$ & $7.54(0.33)_{\mathrm{b}}$ & $12.02(0.31)$ \\
\hline Education & & $t(424.7)=6.16, d=0.58 * *$ & $t(451)=4.43, d=0.26^{* *}$ \\
\hline$>4$ year college $(=0)$ & $163(36.0 \%)$ & $6.09(0.41)_{\mathrm{a}}$ & $10.79(0.42)_{\mathrm{a}}$ \\
\hline College degree or higher $(=1)$ & $290(64.0 \%)$ & $9.72(0.43)_{\mathrm{b}}$ & $13.22(0.34)_{\mathrm{b}}$ \\
\hline Gender & & $F(2452)=11.55, \eta^{2}=0.05 * *$ & $F(2452)=2.27, \eta^{2}=0.01, p=0.10$ \\
\hline Cis woman & $185(40.8 \%)$ & $6.62(0.43)_{\mathrm{a}}$ & $11.83(0.42)$ \\
\hline Cis man & $245(54.1 \%)$ & $9.73(0.46)_{\mathrm{b}}$ & $12.85(0.36)$ \\
\hline Gender minority & $23(5.1 \%)$ & $8.87(1.55)_{\mathrm{b}}$ & $11.09(1.26)$ \\
\hline $\begin{array}{l}\text { Income } \\
(\text { sample median }=50-60,000)\end{array}$ & & $t(451)=1.04, d=0.10, p=0.30$ & $t(451)=3.66, d=0.35^{* *}$ \\
\hline$<50-60,000$ & $245(54 \%)$ & $8.72(6.87)$ & $13.24(5.62)_{\mathrm{a}}$ \\
\hline$\geq 50-60,000$ & $208(46 \%)$ & $8.06(6.74)$ & $11.29(5.68)_{\mathrm{b}}$ \\
\hline
\end{tabular}

Test statistics and effect sizes are bolded for dichotomous comparisons ( $t$-test and ANOVA)

$* * p<0.001$

${ }^{a}$ BMI data were excluded for 18 participants who provided heights/weights that yielded unrealistic values (e.g., BMI < 10.0)

${ }^{\mathrm{b}}$ Means with different subscripts in the same column are significantly different

time, and not related to other health behaviors when adjusting for the TOS subscales (Table 3).

\section{Discussion}

The aims of the current study were to evaluate the factor structure, internal consistency, and convergent validity of an English-language version of the TOS (Aim 1), and to explore the differential relationships between $\mathrm{OrNe}$ and
HeOr and nutrition knowledge, eating behavior, and symptoms of weight/shape disordered eating and other healthrelated behaviors (Aim 2).

\section{Aim 1. Factor structure and convergent validity}

The two-factor structure of the original Spanish TOS was replicated in both samples. However, several items demonstrated cross-loadings in one or both samples, one of which had its strongest loading on a different factor than 


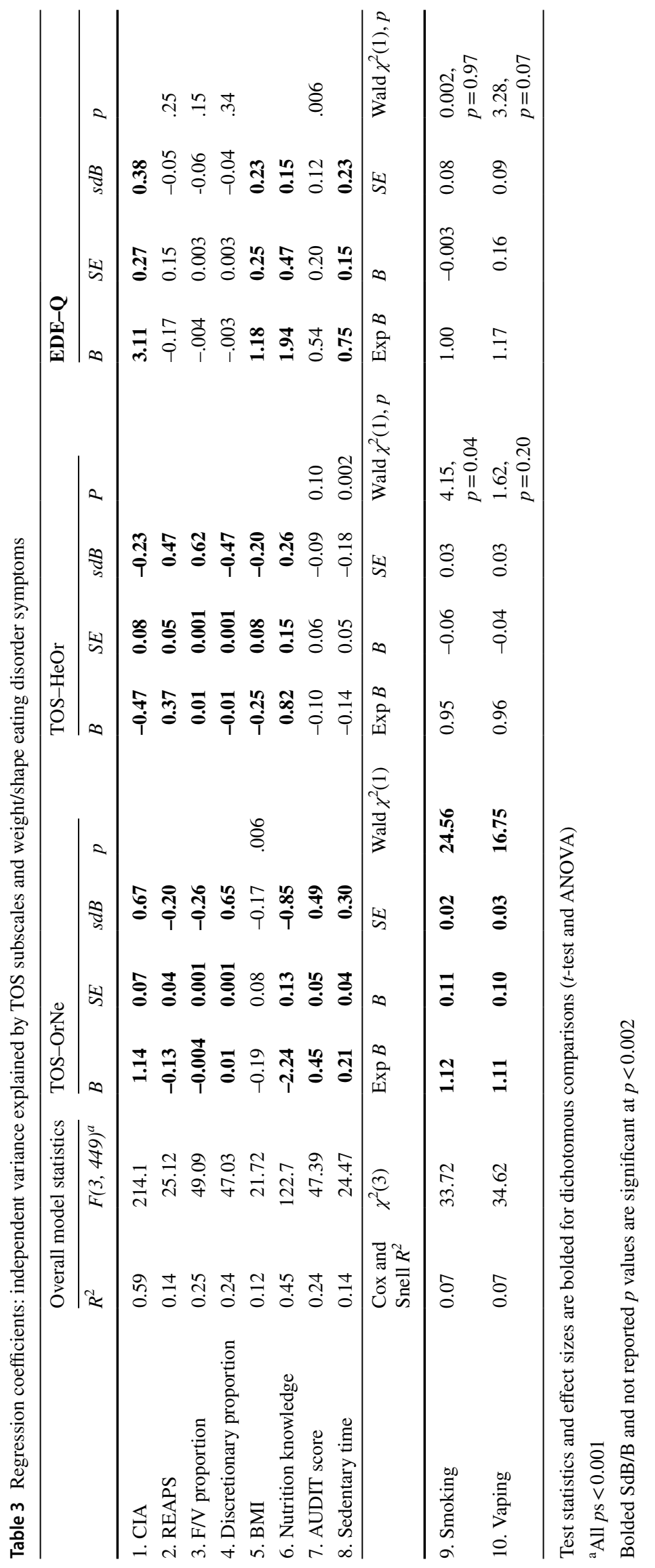


expected in one sample. Because the two-factor structure and original item loadings were replicated in one sample, and to maintain consistency with prior research using the TOS, we chose to proceed with the original item loadings in convergent validity and other correlational analyses.

In both samples the HeOr and OrNe subscales of the TOS were intercorrelated, as predicted. The correlation was stronger in a sample of adults recruited on MTurk $(r=0.60)$ compared to a sample of Yoga practitioners $(r=0.31)$. Results from this study converge with other reports to suggest that $\mathrm{OrNe}$ and $\mathrm{HeOr}$ are distinct latent constructs $[4,18,19]$.

As hypothesized, there was a strong relationship between the TOS-OrNe scale and the DOS total score. Contrary to our hypotheses, however, the relationship between DOS and TOS-HeOr, although attenuated when controlling for TOSOrNe, remained significant with a moderate-large effect size. In the present study, the DOS was chosen as a measure of OrNe; the measure has been shown to have a unidimensional factor structure in several samples [11, 17], but other analyses have suggested the presence of more than one factor [14-16]. One of these unmeasured latent factors might tap a construct closer to $\mathrm{HeOr}$ than OrNe, which the DOS is intended to measure. Given the importance of measuring both $\mathrm{OrNe}$ and HeOr to reveal suppression of relationships with the potentially meaningful clinical outcomes demonstrated in this data and in prior publications [4, 18, 19], identifying, and potentially expanding, a DOS-HeOr factor might improve that measure's clinical and research utility.

\section{Aim 2. Disordered eating symptoms and healthy lifestyle indicators}

As hypothesized, partial correlation analyses revealed suppression effects in the relationship between the TOS subscales and measures of weight/shape disordered eating. Whereas both TOS subscales had moderate, positive zero-order relationships with the EDE-Q, the partial correlation between $\mathrm{HeOr}$ and EDE-Q was small but negative when adjusting for OrNe, whereas the positive relationship between OrNe and EDE-Q was larger than the zero-order effect size when controlling for HeOr. These findings further highlight the need for study designs that statistically adjust for $\mathrm{HeOr}$ when OrNe is the variable of interest.

Overall, Aim 2 results revealed a consistent pattern of correlations, where OrNe was predictive of poorer functioning (e.g., eating disordered clinical impairment), poorer diet quality including greater intake of discretionary foods and lower intake of fruit and vegetables as a proportion of overall diet, poorer nutrition knowledge, more sedentary behavior, more problematic alcohol use, and nicotine use. OrNe was also associated with lower BMI, although the $p$ value associated with this relationship did not meet the
Bonferroni-corrected threshold of $p<0.002$ used in these analyses. HeOr showed the opposite pattern of relationships with eating behavior and nutrition knowledge and was uncorrelated to all other indicators of a healthy lifestyle. This was contrary to the hypothesis that a non-pathological interest in healthy eating would be related to non-dietary indicators of a healthy lifestyle.

Notably, significant relationships between all outcome variables and both TOS subscales were independent of EDE-Q scores. OrNe showed a different pattern of relationships with eating behaviors and some lifestyle variables compared to the EDE-Q. Although related to shape/weight disordered eating, OrNe has independent relationships with disordered eating impairment and is uniquely related to diet quality and eating behavior. These findings offer further support for the conceptualization of OrNe as a distinct eating disorder manifestation and not a presentation of weight/ shape disordered eating.

\section{Strengths and limitations}

Strengths of the manuscript include the use of rigorous psychometric analyses to assess the factor structure of the TOS, and the novelty of Aim 2 analyses exploring the relationships of OrNe and $\mathrm{HeOr}$ with variables measuring eating behavior, nutrition knowledge, and healthy lifestyle behaviors. There were also notable weaknesses, including the use of two non-representative samples of Yoga practitioners and Mechanical Turk workers. Although Mechanical Turk workers are more diverse than the college-student convenience samples in which much of the earlier orthorexia and TOS research has been conducted [2, 18, 19], they have been found to be more highly educated, lower-income, and more psychologically distressed than representative samples in the US [35]. Additional weaknesses specific to Aim 1 are that the version of the English TOS used in both samples was not the same, and that the Yoga instructor data came from international respondents, whereas the MTurk sample was restricted to the US. Further research is needed to assess the factor structure of the English TOS and the cross-cultural measurement invariance of the TOS between English- and Spanish-speaking samples, and of the TOS-En between native and non-native English speakers (who were represented in the Yoga sample in the present study).

Finally, Mturk data for this study were collected in May 2020, at a time in the global Coronavirus-19 (COVID-19) pandemic when most parts of the US were under stay-athome orders. Emerging data suggest that the COVID-19 lockdowns influenced eating, substance use, and exercise behavior in US adults, although findings are decidedly mixed as to the degree or direction of the impact of COVID-19 and the moderators of this relationship [36-41]. Evidence from one large survey of MTurk workers collected before and 
during COVID-19 lockdowns suggests that there may have been influx of new workers to MTurk during the lockdown, a development which appeared to have resulted in more invalid responses identified by attention and validity checks, but more racially and socioeconomically diverse samples than prior to the pandemic [42]. A second study did not find significant differences in the characteristics or experience of workers pre- and post-COVID-19 lockdowns [43]. Clearly, more research is needed to clarify the moderators of COVID-19's influence on eating and health behavior (e.g., pre-COVID-19 mental health symptoms, socioeconomic status, coping strategies, personal impact of the pandemic). In the present sample, the number of workers excluded due to failing attention and validity checks was similar to our previous MTurk samples [e.g., 4]. Given that data were collected during a time of unprecedented national emotional and economic stress and abrupt lifestyle changes whose impact is far from being understood, replication is warranted.

Although not a limitation of the present study, it is important to note that differing patterns of associations between $\mathrm{HeOr}$ and $\mathrm{OrNe}$ and eating disorder symptoms, eating behaviors, diet quality, nutrition knowledge, and lifestyle variables were only apparent, or were more apparent, in regression models estimating independent effects of each scale. The results do not suggest that the clinical presentation of OrNe does not involve healthy eating, or that it involves exclusively unhealthy eating patterns, but rather that $\mathrm{OrNe}$ and $\mathrm{HeOr}$ are separable constructs, that different individuals may exhibit to different degrees.

\section{Conclusions}

Although there does not appear to be a consensus on how OrNe should be defined, most available definitions of the disorder emphasize obsessions, anxiety, and overvalued ideas around an individual's own perception of "healthy eating," but do not require that (1) the patient's definition of "healthy" is consistent with official dietary recommendations or commonly accepted beliefs about healthy eating, or that (2) the individual successfully adheres to a rigid diet [1]. On the latter point, whereas some authors argue that compulsive behaviors related to healthy eating obsessions should be part of the diagnostic picture of OrNe [1], even this definition does not preclude frequent lapses in dietary adherence. People with other restrictive eating disorders (particularly bulimia nervosa and binge-purge anorexia nervosa, but also restrictive anorexia nervosa) struggle to consistently adhere to extreme weight-control diets, and these lapses contribute to the maintenance of symptoms (e.g., inappropriate compensatory behaviors, increased restrictiveness following lapses) and to psychological distress.
Evidence from the current study suggests that, despite reporting intense preoccupation with perceived healthy eating, people with OrNe symptoms engage in eating behavior that is less healthy according to recognized nutrition standards, have lower nutrition literacy, report more sedentary screen time, and are more likely to report smoking, vaping, and problematic alcohol consumption when adjusting for $\mathrm{HeOr}$ and weight/shape eating disorder symptoms. Given the historical problems with measurement validity in the orthorexia literature, we call for continued investigation of the factor structure of the TOSEn, along with that of other commonly used measures. Although there is as yet no clear gold-standard measure of OrNe, we recommend the use of the TOS, and the revision/ expansion of measures like the DOS and EHQ, to assess non-pathological interest and engagement in healthy eating.

\section{What is already known on this subject?}

OrNe is related to symptoms of disordered eating (e.g., shape/weight concerns, restraint) and $\mathrm{OrNe}$ and $\mathrm{HeOr}$ are related but separate constructs.

\section{What does this study add?}

This study is the first to show distinct patterns of associations between $\mathrm{OrNe} / \mathrm{HeOr}$ and indicators of a relatively healthy lifestyle other than psychopathology, BMI, or food choice. This is only the fourth study to explore the relationship between OrNe and specific food choices and/ or diet quality, and the first to use a measure designed to assess $\mathrm{HeOr}$ to examine the specificity of the $\mathrm{OrNe}$ and nutrition relationship.

\section{Appendix A-Teruel Orthorexia Scale}

The following questions relate to the ideas and attitudes you have regarding food. In particular, we would like to know how important it is for you to follow a healthy diet based on foods such as those free of fats, salt, preservatives, additives made by humans or any substance that you consider harmful or toxic, as herbicides or pesticides. 


\begin{tabular}{|c|c|c|c|c|c|c|c|c|c|c|c|}
\hline & & $\begin{array}{l}\text { Com- } \\
\text { pletely } \\
\text { disagree }\end{array}$ & $\begin{array}{l}\text { Slightly } \\
\text { agree }\end{array}$ & $\begin{array}{l}\text { Quite } \\
\text { agree }\end{array}$ & $\begin{array}{l}\text { Com- } \\
\text { pletely } \\
\text { agree }\end{array}$ & & & $\begin{array}{l}\text { Com- } \\
\text { pletely } \\
\text { disagree }\end{array}$ & $\begin{array}{l}\text { Slightly } \\
\text { agree }\end{array}$ & $\begin{array}{l}\text { Quite } \\
\text { agree }\end{array}$ & $\begin{array}{l}\text { Com- } \\
\text { pletely } \\
\text { agree }\end{array}$ \\
\hline 1. & $\begin{array}{l}\text { I feel good } \\
\text { when I eat } \\
\text { healthy } \\
\text { food }\end{array}$ & $\square$ & $\square$ & $\square$ & $\square$ & 8. & $\begin{array}{l}\text { I mainly eat } \\
\text { foods that } \\
\text { I consider } \\
\text { healthy }\end{array}$ & $\square$ & $\square$ & $\square$ & $\square$ \\
\hline \multirow[t]{2}{*}{2.} & \multirow{2}{*}{$\begin{array}{l}\text { I spend a } \\
\text { lot of time } \\
\text { buying, } \\
\text { planning, } \\
\text { and or/ } \\
\text { preparing } \\
\text { food so } \\
\text { my diet } \\
\text { will be as } \\
\text { healthy as } \\
\text { possible }\end{array}$} & \multirow[t]{2}{*}{$\square$} & \multirow[t]{2}{*}{$\square$} & \multirow[t]{2}{*}{$\square$} & \multirow[t]{2}{*}{$\square$} & 9. & $\begin{array}{l}\text { My concern } \\
\text { with } \\
\text { healthy } \\
\text { eating } \\
\text { takes up a } \\
\text { lot of my } \\
\text { time }\end{array}$ & $\square$ & $\square$ & $\square$ & $\square$ \\
\hline & & & & & & 10. & $\begin{array}{l}\text { I am con- } \\
\text { cerned } \\
\text { about the } \\
\text { possibility }\end{array}$ & $\square$ & $\square$ & $\square$ & $\square$ \\
\hline \multirow[t]{2}{*}{3.} & \multirow{2}{*}{$\begin{array}{l}\text { I think that } \\
\text { my way of } \\
\text { eating is } \\
\text { healthier } \\
\text { than that } \\
\text { of most } \\
\text { people }\end{array}$} & \multirow[t]{2}{*}{$\square$} & \multirow[t]{2}{*}{$\square$} & \multirow[t]{2}{*}{$\square$} & \multirow[t]{2}{*}{$\square$} & & $\begin{array}{l}\text { of eating } \\
\text { unhealthy } \\
\text { foods }\end{array}$ & & & & \\
\hline & & & & & & 11. & $\begin{array}{l}\text { I don't mind } \\
\text { spend- } \\
\text { ing more } \\
\text { money on }\end{array}$ & $\square$ & $\square$ & $\square$ & $\square$ \\
\hline \multirow[t]{2}{*}{4.} & \multirow{2}{*}{$\begin{array}{l}\text { I feel guilty } \\
\text { when I eat } \\
\text { food that } \\
\text { I do not } \\
\text { consider } \\
\text { healthy }\end{array}$} & \multirow[t]{2}{*}{$\square$} & \multirow[t]{2}{*}{$\square$} & \multirow[t]{2}{*}{$\square$} & \multirow[t]{2}{*}{$\square$} & & $\begin{array}{l}\text { a food if I } \\
\text { think it's } \\
\text { healthier }\end{array}$ & & & & \\
\hline & & & & & & 12. & $\begin{array}{l}\text { I feel over- } \\
\text { whelmed } \\
\text { or sad if }\end{array}$ & $\square$ & $\square$ & $\square$ & $\square$ \\
\hline \multirow[t]{2}{*}{5.} & \multirow{2}{*}{$\begin{array}{l}\text { My social } \\
\text { relation- } \\
\text { ships } \\
\text { have been } \\
\text { negatively } \\
\text { affected by } \\
\text { my con- } \\
\text { cern about } \\
\text { eating } \\
\text { healthy } \\
\text { food }\end{array}$} & \multirow[t]{2}{*}{$\square$} & \multirow[t]{2}{*}{$\square$} & \multirow[t]{2}{*}{$\square$} & \multirow[t]{2}{*}{$\square$} & & $\begin{array}{l}\text { I eat food } \\
\text { that I } \\
\text { consider } \\
\text { unhealthy }\end{array}$ & & & & \\
\hline & & & & & & 13. & $\begin{array}{l}\text { I would } \\
\text { rather eat } \\
\text { a smaller } \\
\text { portion of } \\
\text { healthy } \\
\text { food than } \\
\text { get full }\end{array}$ & $\square$ & $\square$ & $\square$ & $\square$ \\
\hline \multirow[t]{2}{*}{6.} & \multirow{2}{*}{$\begin{array}{l}\text { My interest } \\
\text { in healthy } \\
\text { food is an } \\
\text { important } \\
\text { part of the } \\
\text { way I am, } \\
\text { of how I } \\
\text { understand } \\
\text { the world }\end{array}$} & \multirow[t]{2}{*}{$\square$} & \multirow[t]{2}{*}{$\square$} & \multirow[t]{2}{*}{$\square$} & \multirow[t]{2}{*}{$\square$} & & $\begin{array}{l}\text { from food } \\
\text { that may } \\
\text { not be } \\
\text { healthy }\end{array}$ & & & & \\
\hline & & & & & & 14. & $\begin{array}{l}\text { I avoid eat- } \\
\text { ing with } \\
\text { people } \\
\text { who do } \\
\text { not share }\end{array}$ & $\square$ & $\square$ & $\square$ & $\square$ \\
\hline \multirow[t]{2}{*}{7.} & \multirow{2}{*}{$\begin{array}{l}\text { I'd rather eat } \\
\text { a healthy } \\
\text { food that } \\
\text { is not very } \\
\text { tasty than } \\
\text { a good- } \\
\text { tasting } \\
\text { food } \\
\text { that isn't } \\
\text { healthy }\end{array}$} & \multirow[t]{2}{*}{$\square$} & \multirow[t]{2}{*}{$\square$} & \multirow[t]{2}{*}{$\square$} & \multirow[t]{2}{*}{$\square$} & & $\begin{array}{l}\text { my ideas } \\
\text { about } \\
\text { healthy } \\
\text { eating }\end{array}$ & & & & \\
\hline & & & & & & 15. & $\begin{array}{l}\text { I try to } \\
\text { convince } \\
\text { the people } \\
\text { in my life } \\
\text { to follow } \\
\text { my healthy }\end{array}$ & $\square$ & $\square$ & $\square$ & $\square$ \\
\hline
\end{tabular}




\begin{tabular}{|c|c|c|c|c|c|}
\hline & & $\begin{array}{l}\text { Com- } \\
\text { pletely } \\
\text { disagree }\end{array}$ & $\begin{array}{l}\text { Slightly } \\
\text { agree }\end{array}$ & $\begin{array}{l}\text { Quite } \\
\text { agree }\end{array}$ & $\begin{array}{l}\text { Com- } \\
\text { pletely } \\
\text { agree }\end{array}$ \\
\hline 16. & $\begin{array}{l}\text { If I ever eat } \\
\text { something } \\
\text { I consider } \\
\text { unhealthy, } \\
\text { I punish } \\
\text { myself } \\
\text { for it }\end{array}$ & $\square$ & $\square$ & $\square$ & $\square$ \\
\hline 17. & $\begin{array}{l}\text { Thoughts } \\
\text { about } \\
\text { healthy } \\
\text { eating } \\
\text { prevent me } \\
\text { from con- } \\
\text { centrating } \\
\text { on other } \\
\text { tasks }\end{array}$ & $\square$ & $\square$ & $\square$ & $\square$ \\
\hline
\end{tabular}

\section{Scoring}

Completely disagree $=0$; Slightly agree $=1$; Quite agree $=2$; Strongly agree $=3$.

TOS Healthy Orthorexia $=$ TOS $1+$ TOS $2+$ TOS $3+$ TOS $6+\mathrm{TOS} 7+\mathrm{TOS} 8+\operatorname{TOS} 11+\mathrm{TOS} 13+\mathrm{TOS} 15$.

TOS Orthorexia Nervosa $=$ TOS $4+$ TOS $5+$ TOS $9+$ TOS $10+\operatorname{TOS} 12+\mathrm{TOS} 14+\mathrm{TOS} 16+\mathrm{TOS} 17$.

\section{Declarations}

Conflict of interest The authors have no conflicts of interest to report.

Ethical approval The studies were approved by the authors' university IRBs.

Informed consent All participants provided informed consent.

\section{References}

1. Cena H, Barthles F, Cuzzolaro M, Bratman S, Brytek-Matera A, Dunn T, Donini LM (2019) Definition and diagnostic criteria for orthorexia nervosa: a narrative review of the literature. Eat Weight Disord 24(2):209-246. https://doi.org/10.1016/j.eatbeh.2015.12. 006

2. Barthles F, Barrada JR, Roncero M (2019) Orthorexia nervosa and healthy orthorexia as new eating styles. PLoS ONE 14(7):e0219609. https://doi.org/10.1371/journal.pone.0219609

3. Stutts LA (2020) It's complicated: The relationship between orthorexia and weight/shape concerns, eating behaviors, and mood. Eat Behav 39:101444. https://doi.org/10.1016/j.eatbeh.2020.101444

4. Zickgraf HF, Ellis JM, Essayli JH (2019) Disentangling orthorexia nervosa from healthy eating and other eating disorder symptoms: Relationships with clinical impairment, comorbidity, and self-reported food choices. Appetite 134:40-49. https://doi.org/ 10.1016/j.appet.2018.12.006

5. Bartel SJ, Sherry SB, Farthing GR, Stewart SH (2020) Classification of orthorexia nervosa: further evidence for placement within the eating disorders spectrum. Eat Behav 38:101406. https://doi. org/10.1016/j.eatbeh.2020.101406

6. Strahler J, Stark R (2020) Perspective: classifying orthorexia nervosa as a new mental illness-much discussion, little evidence. Adv Nutr 11(4):784-789. https://doi.org/10.1093/advances/nmaa0 12

7. Gleaves DH, Graham EC, Ambwani S (2013) Measuring "orthorexia": development of the eating habits questionnaire. Int J Edu Psychol Assess. https://doi.org/10.3390/nu12123820

8. Barthles F, Meyer F, Pietrowsky R (2015) Die düsseldorfer orthorexie Skala-Konstruktion und evaluation eines fragebogens zur erfassung ortho-rektischen ernährungsverhaltens. Z Klin Psychol Psychother. https://doi.org/10.1026/1616-3443/a000310

9. Godefroy V, Trinchera L, Dorard G (2021) Optimizing the empirical assessment of orthorexia nervosa through EHQ and clarifying its relationship with BMI. Eat Weight Disord 26(2):649-659. https://doi.org/10.1007/s40519-020-00909-4

10. Halim ZM, Dickinson KM, Kemps E, Prichard I (2020) Orthorexia nervosa: Examining the Eating Habits Questionnaire's reliability and validity, and its links to dietary adequacy among adult women. Public Health Nutr 23(10):1684-1692. https://doi. org/10.1017/s1368980019004282

11. Meule A, Holzapfel C, Brandl B, Greetfeld M, Hessler-Kaufmann JB, Skurk T, Voderholzer U (2020) Measuring orthorexia nervosa: a comparison of four self-report questionnaires. Appetite 146:104512. https://doi.org/10.1016/j.appet.2019.104512

12. Oberle CD, Samaghabadi RO, Hughes EM (2017) Orthorexia nervosa: assessment and correlates with gender, BMI, and personality. Appetite 108:303-310. https://doi.org/10.1016/j.appet. 2016.10.021

13. Yakın E, Raynal P, Chabrol H (2020) Distinguishing orthorexic behaviors from eating disordered and obsessive-compulsive behaviors: a typological study. Eat Weight Disord 1:9. https:// doi.org/10.1007/s40519-020-01037-9

14. Chard CA, Hilzendegen C, Barthles F, Stroebele-Benschop N (2019) Psychometric evaluation of the English version of the Düsseldorf Orthorexie Scale (DOS) and the prevalence of orthorexia nervosa among a US student sample. Eat Weight Disord 24(2):275-281. https://doi.org/10.1007/s40519-018-0570-6

15. Lasson C, Barthles F, Raynal P (2021) Psychometric evaluation of the French version of the Düsseldorfer Orthorexia Skala (DOS) and prevalence of orthorexia nervosa among university students. Eat Weight Disord. 1-8. https://doi.org/10.1007/ s40519-021-01123-6

16. He J, Ma H, Barthles F, Fan X (2019) Psychometric properties of the Chinese version of the Düsseldorf Orthorexia Scale: prevalence and demographic correlates of orthorexia nervosa among Chinese university students. Eat Weight Disord 24(3):453-463. https://doi.org/10.1007/s40519-019-00656-1

17. Parra-Fernández ML, Onieva-Zafra MD, Fernández-Muñoz JJ, Fernández-Martínez E (2019) Adaptation and validation of the Spanish version of the DOS questionnaire for the detection of orthorexic nervosa behavior. PLoS ONE 14(5):e0216583. https://doi.org/10.1371/journal.pone.0216583

18. Barrada JR, Roncero M (2018) Bidimensional structure of the orthorexia: development and initial validation of a new instrument. Anales De Psicología 34(2):283-291. https://doi.org/10. 6018/analesps.34.2.299671

19. Depa J, Barrada JR, Roncero M (2019) Are the motives for food choices different in orthorexia nervosa and healthy orthorexia? Nutrients 11(3):697. https://doi.org/10.3390/nu11030697 
20. Domingues RB, Carmo C (2020) Orthorexia nervosa in yoga practitioners: relationship with personality, attitudes about appearance, and yoga engagement. Eat Weight Disord 1:7. https://doi.org/10.1007/s40519-020-00911-w

21. Strahler J, Haddad C, Salameh P, Sacre H, Obeid S, Hallit S (2020) Cross-cultural differences in orthorexic eating behaviors: associations with personality traits. Nutrition 77:110811. https://doi.org/10.1016/j.nut.2020.110811

22. Barthles F, Amrhein J, Scharmach K, Meyer F, Pietrowsky R (2018) Konstruktvalidität der Düsseldorfer Orthorexie Skala: Korrelationen mit der gesundheitlichen Qualität des Essverhaltens und essstörungsspezifischen Aspekten [Concurrent construct validity of the düsseldorf orthorexia scale: correlations with diet quality and specific dimensions of disordered eating behavior]. Z Klin Psychol Psychother 47:109-118. https://doi. org/10.1026/1616-3443/a000479

23. Strahler J, Hermann A, Walter B, Stark R (2018) Orthorexia nervosa: a behavioral complex or a psychological condition? J Behav Addict 7(4):1143-1156. https://doi.org/10.1556/2006.7. 2018.129

24. Grilo CM, Reas DL, Hopwood CJ, Crosby RD (2015) Factor structure and construct validity of the Eating Disorder Examination-Questionnaire in college students: Further support for a modified brief version. Int J Eat Disord 48(3):284-289. https:// doi.org/10.1002/eat.22358

25. Fairburn CG, Beglin SJ (2008) Eating disorder examination questionnaire. Cogn Behav Therapy Eat Disord 309:313. https://doi. org/10.1037/t03974-000

26. Bohn K, Fairburn CG (2008) The clinical impairment assessment questionnaire (CIA). Cognit Behav Therapy Eat Disord 315:317. https://doi.org/10.1093/oxfordhb/9780195373622.013.0019

27. Wildes JE, Zucker NL, Marcus MD (2012) Picky eating in adults: results of a web-based survey. Int J Eat Disord 45(4):575-582. https://doi.org/10.1002/eat.20975

28. Segal-Isaacson CJ, Wylie-Rosett J, Gans KM (2004) Validation of a short dietary assessment questionnaire: the Rapid Eating and Activity Assessment for Participants short version (REAPS). Diabetes Educ 30(5):774-781. https://doi.org/10.1177/01457 2170403000512

29. Johnston CS, Bliss C, Knurick JR, Scholtz C (2018) Rapid eating assessment for Participants [shortened version] scores are associated with Healthy Eating Index-2010 scores and other indices of diet quality in healthy adult omnivores and vegetarians. Nutr J 17(1):1-7. https://doi.org/10.1186/s12937-018-0399-x

30. Zickgraf HF, Schepps K (2016) Fruit and vegetable intake and dietary variety in adult picky eaters. Food Qual Prefer 54:39-50

31. Kliemann N, Wardle J, Johnson F, Croker H (2016) Reliability and validity of a revised version of the general nutrition knowledge questionnaire. Eur J Clin Nutr 70(10):1174-1180

32. Bush K, Kivlahan DR, McDonell MB, Fihn SD, Bradley KA (1998) The AUDIT alcohol consumption questions (AUDIT-C): an effective brief screening test for problem drinking. Arch Intern
Med 158(16):1789-1795. https://doi.org/10.1001/archinte.158.16. 1789

33. Wijndaele K, De Bourdeaudhuij I, Godino JG, Lynch BM, Griffin SJ, Westgate K, Brage S (2014) Reliability and validity of a domain-specific last 7-d sedentary time questionnaire. Med Sci Sports Exerc 46(6):1248. https://doi.org/10.1249/mss.0000000000 000459

34. Asparouhov T, Muthén B (2009) Exploratory structural equation modeling. Struct Equ Model 16:397-438. https://doi.org/10.1080/ 10705510903008204

35. Hu L, Bentler PM (1999) Cutoff criteria for fit indexes in covariance structure analysis: Conventional criteria versus new alternatives. Struct Equ Model 6:1-55. https://doi.org/10.1080/10705 519909540118

36. Xia Y, Yang Y (2019) RMSEA, CFI, and TLI in structural equation modeling with ordered categorical data: the story they tell depends on the estimation methods. Behav Res Methods 51:409 428. https://doi.org/10.3758/s13428-018-1055-2

37. Arditte KA, Çek D, Shaw AM, Timpano KR (2016) The importance of assessing clinical phenomena in Mechanical Turk research. Psychol Assess 28(6):684. https://doi.org/10.1037/pas00 00217

38. Cummings JR, Ackerman JM, Wolfson JA, Gearhardt AN (2021) COVID-19 stress and eating and drinking behaviors in the United States during the early stages of the pandemic. Appetite 162:105163. https://doi.org/10.1016/j.appet.2021.105163

39. Flanagan EW, Beyl RA, Fearnbach SN, Altazan AD, Martin CK, Redman LM (2021) The impact of COVID-19 stay-at-home orders on health behaviors in adults. Obesity 29(2):438-445. https://doi.org/10.1002/oby.23066

40. Knell G, Robertson MC, Dooley EE, Burford K, Mendez KS (2020) Health behavior changes during COVID-19 pandemic and subsequent "stay-at-home" orders. Int J Environ Res Public Health 17(17):6268. https://doi.org/10.3390/ijerph17176268

41. Mason TB, Barrington-Trimis J, Leventhal AM (2021) Eating to cope with the COVID-19 pandemic and body weight change in young adults. J Adolesc Health 68(2):277-283. https://doi.org/10. 1016/j.jadohealth.2020.11.011

42. Arechar AA, Rand DG (2021) Turking in the time of COVID. Behav Res Methods 1:5. https://doi.org/10.3758/ s13428-021-01588-4

43. Moss AJ, Rosenzweig C, Robinson J, Litman L (2020) Demographic stability on Mechanical Turk despite COVID-19. Trends Cogn Sci 24(9):678-680. https://doi.org/10.1016/j.tics.2020.05. 014

Publisher's Note Springer Nature remains neutral with regard to jurisdictional claims in published maps and institutional affiliations. 